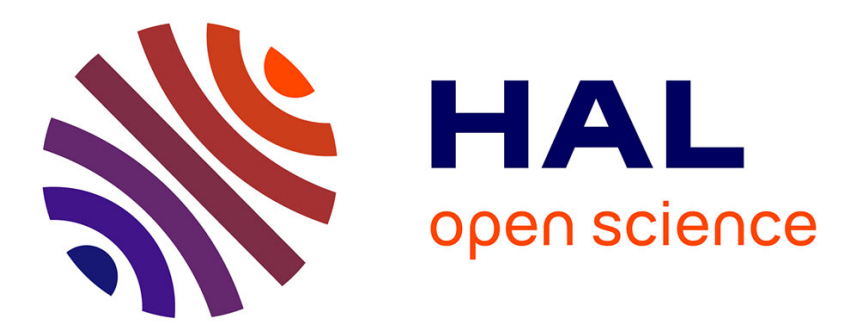

\title{
Electric-field frequency dependence of Pockels coefficients in 2-amino-5-nitropyridium dihydrogen phosphate organic-inorganic crystals
}

Julien Zaccaro, Jean-Paul Salvestrini, A. Ibanez, P. Ney, M. D. Fontana

\section{To cite this version:}

Julien Zaccaro, Jean-Paul Salvestrini, A. Ibanez, P. Ney, M. D. Fontana. Electric-field frequency dependence of Pockels coefficients in 2-amino-5-nitropyridium dihydrogen phosphate organicinorganic crystals. Journal of the Optical Society of America B, 2000, 17 (3), pp.427-432. 10.1364/JOSAB.17.000427 . hal-00186035

\section{HAL Id: hal-00186035 \\ https://hal.science/hal-00186035}

Submitted on 12 Jan 2022

HAL is a multi-disciplinary open access archive for the deposit and dissemination of scientific research documents, whether they are published or not. The documents may come from teaching and research institutions in France or abroad, or from public or private research centers.
L'archive ouverte pluridisciplinaire HAL, est destinée au dépôt et à la diffusion de documents scientifiques de niveau recherche, publiés ou non, émanant des établissements d'enseignement et de recherche français ou étrangers, des laboratoires publics ou privés.

\section{(ㄷ)(1) $\$$}

Distributed under a Creative Commons Attribution - NonCommerciall 4.0 International 


\title{
Electric-field frequency dependence of Pockels coefficients in 2-amino-5-nitropyridium dihydrogen phosphate organic-inorganic crystals
}

\author{
J. Zaccaro \\ Laboratoire de Cristallographie Associé à l'Université J. Fourier (Grenoble I), Centre National de la Recherche \\ Scientifique, B.P. 166, 38042 Grenoble Cedex 09, France \\ J. P. Salvestrini \\ Laboratoire Matériaux Optiques à Propriétés Spécifiques, Centre Lorrain d'Optique et Electronique des Solides, \\ Université de Metz et Supelec, 2 Rue E. Belin, 57070 Metz, France
}

A. Ibanez

Laboratoire de Cristallographie Associé à l'Université J. Fourier (Grenoble I), Centre National de la Recherche Scientifique, B.P. 166, 38042 Grenoble Cedex 09, France

P. Ney and M. D. Fontana

Laboratoire Matériaux Optiques à Propriétés Spécifiques, Centre Lorrain d'Optique et Electronique des Solides, Université de Metz de Supelec, 2 Rue E. Belin, 57070 Metz, France

\begin{abstract}
The electro-optic and dielectric properties of organic-inorganic 2-amino-5-nitropyridium dihydrogen phosphate crystals were measured near room temperature at a wavelength of $632.8 \mathrm{~nm}$ and as a function of an electric-field frequency that v aried from $1 \mathrm{kHz}$ to $40 \mathrm{M} \mathrm{Hz}$. The frequency dispersion of the electro-optic coefficients and their comparison with the second-harmonic generation coefficients showed that the largest part of the electro-optic properties is electronic in origin (whereas the piezo-optic contribution is small). Results reveal a large anisotropy of the ionic and electronic contributions that is related to the contributions of the organic molecule to nonlinear polarizability.
\end{abstract}

\section{INTRODUCTION}

It is now well established that organic materials possess large nonlinear optical (NLO) coefficients, and this property renders them promising for applications in wave mixing. ${ }^{1}$ However, unlike to inorganic materials, ${ }^{2}$ organic materials generally have low resistance to optical and mechanical damage. This drawback can be reduced by use of a combination of organic and inorganic components to form an organic-inorganic crystalline structure. This new approach can be an important way to meet the challenge of finding new hybrid NLO materials with improved mechanical and thermal properties. ${ }^{3}$ Within this strategy, it was recently demonstrated that 2-amino-5nitropyridium dihydrogen phosphate (2A5NPDP) and 2-amino-5-nitropyridium dihydrogen arsenate can be interesting crystals with relatively important NLO coefficients. $^{4,5}$

To optimize these hybrid materials it can be useful to estimate the respective contributions of the mineral and organic groups to the nonlinear susceptibility. Thus we aim to link the electro-optic (EO) and the secondharmonic generation (SHG) coefficients according to the classic analysis proposed by Sigelles and Hierle. ${ }^{6}$ We thus derive the frequency dependence of the quadratic nonlinear susceptibility $\chi^{(2)}$.

Because it is generally recognized that in organic crystals the EO coefficients are mainly of electronic origin ${ }^{2}$ and equivalently that $\chi^{(2)}$ is frequency independent, any frequency dependence of $\chi^{(2)}$ at low frequency in organicinorganic crystals can reasonably be attributed to the mineral subnetwork. We have therefore undertaken measurements of the EO coefficients as a function of the modulating field frequency to compare them with the SHG coefficients that provide the purely electronic contributions to $\chi^{(2)}$. All these results are interpreted in terms of ionic and electronic contributions or mineral and organic group influences. The special consequence for anisotropy of the properties is also discussed.

\section{THEORETICAL BACKGROUND}

Second-order susceptibility can be defined as the nonlinear mixing of two monochromatic waves with different frequencies $\omega_{1}$ and $\omega_{2}$ to induce a third wave at a frequency $\omega_{3}=\omega_{1}+\omega_{2}$. 
If one of the two mixing waves has a frequency $\omega_{2}$ that is largely smaller (radio frequency) than the other one, $\omega_{1}=\Omega$ (optical frequency), we obtain the EO susceptibility as $\chi_{i j k}^{(2)}(\Omega ; \Omega ; 0)$, which corresponds to the electronic contribution of the EO (Pockels) coefficient as

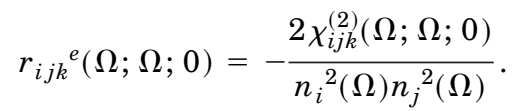

Thus the electronic EO coefficient $r_{i j k}{ }^{e}$ can be compared with the SHG coefficient $d_{i j k}(2 \omega, \omega, \omega)=1 / 2 \chi_{i j k}^{(2)}(2 \omega$; $\omega ; \omega)$ as

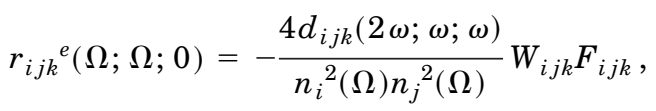

where two correction factors have been introduced. $W_{i j k}$ accounts for the medium dispersion:

$$
W_{i j k}=\frac{\left[\omega_{0}^{2}-(2 \omega)^{2}\right]_{i}\left(\omega_{0}^{2}-\omega^{2}\right)_{j}\left(\omega_{0}^{2}-\omega^{2}\right)_{k}}{\left(\omega_{0}^{2}-\Omega^{2}\right)_{i}\left(\omega_{0}^{2}-\Omega^{2}\right)_{j} \omega_{0 k}{ }^{2}},
$$

where $\omega_{0}$ is the main absorption frequency and $F_{i j k}$ is the local field correction:

$$
F_{i j k}=\frac{f_{\Omega, i} f_{\Omega, j} f_{0, k}}{f_{2 \omega, i} f_{\omega, j} f_{\omega, k}} .
$$

Within the Onsager model we have $f_{\omega, i}=\epsilon_{\omega, i}\left(\epsilon_{\infty, i}\right.$ $+2) /\left(2 \epsilon_{\omega, i}+\epsilon_{\infty, i}\right)$ where $\epsilon_{\infty}$ is the value of the highfrequency limit of the squared refractive index. Recall that $\Omega$ is the laser frequency used in the EO measurements, whereas $\omega$ is the fundamental wave frequency employed in the SHG experiment.

At low frequency, other contributions can be added to the electronic EO coefficient. They arise from indirect modulation of the susceptibility by means of the ionic lattice and, if the crystal is piezoelectric, by means of crystal deformation. ${ }^{7}$ The total EO coefficient $r^{T}$ for a crystal that is free of any stress can be then written as

$$
r_{i j k}^{T}=r_{i j k}{ }^{s}+p_{i j m n} D_{m n k},
$$

where $D_{m n k}$ and $p_{i j m n}$ are the piezoelectric and elastooptic coefficients, respectively, and $r^{s}$ is the EO coefficient measured in the clamped crystal, above the piezoelectric resonances. The product $\left(p_{i j m n} D_{m n k}\right)$ is hereafter called the piezo-optic contribution of the EO coefficient, $r_{i j k}{ }^{\text {po }}$. One can use the dependence of $r_{i j k}$ on frequency from 0 to several megahertz to separate the clamped (ionic + electronic) contribution from the piezo-optic effect. The comparison of $r_{i j k}{ }^{s}$ and $r_{i j k}{ }^{e}$ given by Eq. (2) allows us to estimate the ionic and electronic contributions. Therefore the total (low-frequency) EO coefficient $r^{T}$ can be written as

$$
r_{i j k}^{T}=r_{i j k}^{\text {po }}+r_{i j k}^{i}+r_{i j k}^{e},
$$

where $r_{i j k}{ }^{i}$ is the ionic contribution of the EO Pockels coefficient.

\section{DESCRIPTION OF THE CRYSTAL}

The structure of the 2A5NPDP crystal is based on the adhesion of organic molecules that exhibit a large NLO efficiency to mineral layers whose presence ensures conve-

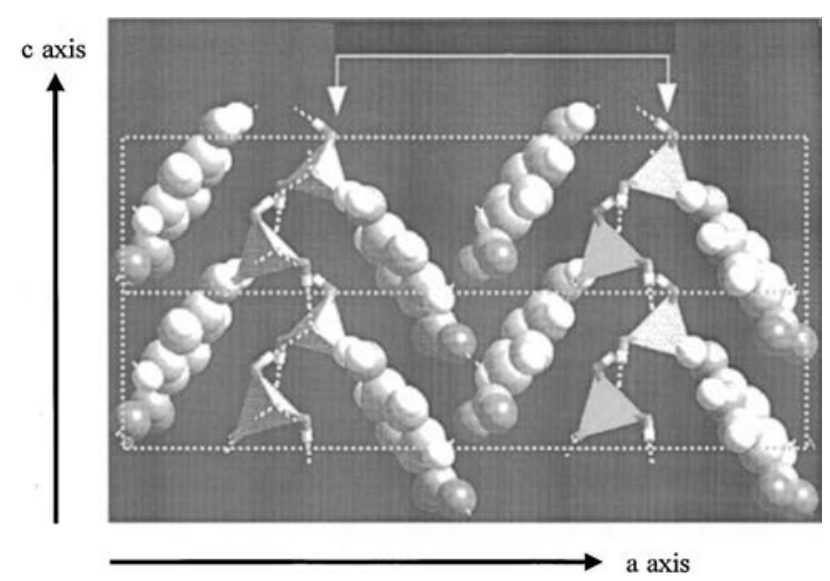

Fig. 1. Chemical structure of the organic-inorganic 2A5NPDP crystal. The arrows indicate the mineral plane $\left(\mathrm{H}_{2} \mathrm{PO}_{4}{ }^{-}\right)$.

nient thermal and mechanical stability and also a high threshold to optical damage. The synthesis and crystal growth of these organic-inorganic phases are now well controlled. $^{8,9}$ At room temperature the crystal structure has orthorhombic $\mathrm{mm} 2$ symmetry. The mineral subnetwork is built up from polar $\left[\mathrm{H}_{2} \mathrm{PO}_{4}{ }^{-}\right]_{n}$ layers parallel to the $(b, c)$ plane. The organic molecules are anchored to these planes through three short hydrogen bonds in a herringbone pattern (Fig. 1). The axis of the molecules mainly lies in the $(a, c)$ plane, with a reduced angle $\left(\approx 36^{\circ}\right)$ with respect to the twofold $c$ axis. ${ }^{10}$

This 2A5NPDP hybrid salt is synthesized from dissolution of the 2-amino-5-nitropyridium molecule in an aqueous $\mathrm{H}_{3} \mathrm{PO}_{4}$ acidic solution. Its crystal growth occurs in solution by the standard temperature-reduction method. ${ }^{8}$ Large (several square centimeters) and optically clear crystals have been grown in this way. Their good crystal quality was confirmed by x-ray diffraction topography. The improved mechanical stability of these hybrid crystals compared with the corresponding organic molecular crystals (Vickers hardness, $\approx 100$, compared with $\sim 10$ for organics $^{11}$ ) enables one to cut and polish the crystals without any difficulty.

\section{ELECTRO-OPTIC MEASUREMENTS}

\section{A. Electro-Optic Properties}

The linear EO properties of the $m m 2$ orthorhombic crystals are described in an abbreviated notation by five coefficients, $r_{13}, r_{23}, r_{33}, r_{42}$, and $r_{51}$. If the light beam is propagating along the $x_{1}$ axis, the $x_{2}$ axis, or the $x_{3}$ axis and the electrical field is applied along the $x_{3}$ axis, the field-induced birefringence in the $\left(x_{2}, x_{3}\right),\left(x_{1}, x_{3}\right)$, and $\left(x_{1}, x_{2}\right)$ planes is given, respectively, by

$$
\begin{aligned}
& \Delta n_{23}(E)=-\frac{n_{2}{ }^{3} r_{a} E_{3}}{2}, \\
& \Delta n_{13}(E)=-\frac{n_{1}{ }^{3} r_{b} E_{3}}{2}, \quad \Delta n_{12}(E)=-\frac{n_{1}{ }^{3} r_{c} E_{3}}{2},
\end{aligned}
$$

where the effective coefficients $r_{a}, r_{b}$, and $r_{c}$ are defined by 


$$
\begin{aligned}
& r_{a}=r_{23}-\left(n_{3}{ }^{3} / n_{2}{ }^{3}\right) r_{33}, \quad r_{b}=r_{13}-\left(n_{3}{ }^{3} / n_{1}{ }^{3}\right) r_{33}, \\
& r_{c}=r_{13}-\left(n_{2}{ }^{3} / n_{1}{ }^{3}\right) r_{23} .
\end{aligned}
$$

The EO coefficient $r_{42}$ can be determined when the electric field is applied along the $x_{2}$ axis and the light beam is propagating along the $x_{2}{ }^{\prime}$ axis. This new coordinate system $\left(x_{2}{ }^{\prime}, x_{3}{ }^{\prime}\right)$ is obtained by a $45^{\circ}$ rotation of the principal axes about $x_{1}$. Thus the coefficient $r_{42}$ is related to the field-induced birefringence by

$$
\Delta n_{13^{\prime}}(E)=-\frac{n_{23}{ }^{3} r_{42} E_{2}}{2}, \quad n_{23}=\left(\frac{2 n_{2}{ }^{2} n_{3}{ }^{2}}{n_{2}{ }^{2}+n_{3}{ }^{2}}\right)^{1 / 2} .
$$

In the same way, the EO coefficient $r_{51}$ can be obtained when the electric field is applied along the $x_{1}$ axis and the light is propagating along the $x_{1}{ }^{\prime \prime}$ axis, which corresponds to the $x_{1}$ axis rotated at $45^{\circ}$ about the $x_{2}$ axis:

$$
\Delta n_{23^{\prime \prime}}(E)=-\frac{n_{13}{ }^{3} r_{51} E_{1}}{2}, \quad n_{13}=\left(\frac{2 n_{1}{ }^{2} n_{3}{ }^{2}}{n_{1}{ }^{2}+n_{3}{ }^{2}}\right)^{1 / 2} .
$$

Finally, the field-induced phase shift for each component of the light polarization can be expressed by

$$
\Gamma(E)=\frac{2 \pi L}{\lambda} \Delta n(E),
$$

where $L$ is the crystal length along the light-propagation direction, $\lambda$ is the wavelength of the light beam, and $\Delta n(E)$ is the field-induced birefringence in the light's path.

\section{B. Experimental Techniques}

The coefficients $r_{a}, r_{b}, r_{c}, r_{42}$, and $r_{51}$ were determined by means of the ac modulation method with the Sénarmont setup. ${ }^{7,12}$ We measured the frequency dispersions of the $r_{a}$ and $r_{b}$ coefficients, using the 632.8-nm wavelength of a $\mathrm{He}-\mathrm{Ne}$ laser, for an ac modulation voltage with $130-\mathrm{V}$ amplitude and frequencies that varied from 1 $\mathrm{kHz}$ to $1 \mathrm{MHz}$. The EO coefficients $r_{c}, r_{42}$, and $r_{51}$ were determined only at one frequency $(1 \mathrm{kHz})$ because their values are relatively small. The EO coefficients were derived from

$$
r=\frac{\lambda d m}{\pi L n^{3} V}
$$

where $m$ is the modulation coefficient (ratio between the modulated output and the input signal intensities), $d$ is the interelectrode spacing, and $V$ is the amplitude of the applied voltage.

We measured separately the EO coefficients $r_{13}, r_{23}$, and $r_{33}$ by means of an interferometric setup, using a $\mathrm{He}-\mathrm{Ne}$ laser $(\lambda=632.8 \mathrm{~nm})$ as the light source. The crystal under study and an ammonium dihydrogen phosphate based EO modulator were put into the arms of a Michelson interferometer.

The experimental method is based on the compensation, with the EO modulator, of the optical path-length shift that is due to the application of a static electric field to the surface of the crystal.
This static electric field induces a change $\Delta \delta$ in the optical path length $\delta$, which is equal to

$$
\begin{aligned}
\Delta \delta= & 2 \Delta\left(n_{i j} L\right)=2 L\left(-(1 / 2) n_{i j}{ }^{3} r_{i j k}\right. \\
& \left.+\left[n_{i j}-1\right] D_{i j k}\right) E_{k},
\end{aligned}
$$

where $L$ is the crystal's length along the propagation direction of the light beam, $D_{i j k}$ are the piezoelectric coefficients, and $E_{k}$ is the applied electric field.

To compare optical path lengths it is first necessary to make a vertical slit in a fringe at the extreme intensities of the interference [fully constructive $\left(I=I_{\max }\right)$ or fully destructive $\left(I=I_{\min }\right)$. To do this we applied a sinuswave voltage at frequency $\omega$ to the modulator to modulate the phase of the laser beam in the interferometer's arm. At minimum or maximum intensity of interference, the frequency of the output wave is twice $(2 \omega)$ that of the applied electric field, and the signal shape is symmetric. When the interference pattern is shifted, the $2 \omega$ signal disappears. Conditions under which this symmetrical shape of the $2 \omega$ modulation signal is observed are quite sensitive and allow the fringe (dark or clear) to be detected precisely, which is the starting point of all measurements.

If we denote constant $C_{m}$ as

$$
C_{m}=\frac{\lambda}{2 V_{\pi / 2}}
$$

where $\lambda$ and $V_{\pi / 2}$ are the wavelength and the quarterwave voltage of the modulator, respectively, the direct experimental value EO coefficient of the crystal is given by

$$
r_{i j k}=-\frac{e}{n_{i j}{ }^{3} L V} C_{m} \Delta V+2 \frac{\left(n_{i j}-1\right)}{n_{i j}{ }^{3}} D_{i j k} .
$$

The second term on the right-hand side of Eq. (15) corresponds to the contribution of the inverse piezoelectric effect. $n_{i j}, e, L, V$, and $D_{i j k}$ are the refractive index, the thickness in the direction of the applied electric field, the length of the optical path in the sample, the applied voltage, and the piezoelectric coefficients, respectively. $\Delta V$ is the voltage needed to counterbalance the effect of the dc voltage applied to the sample.

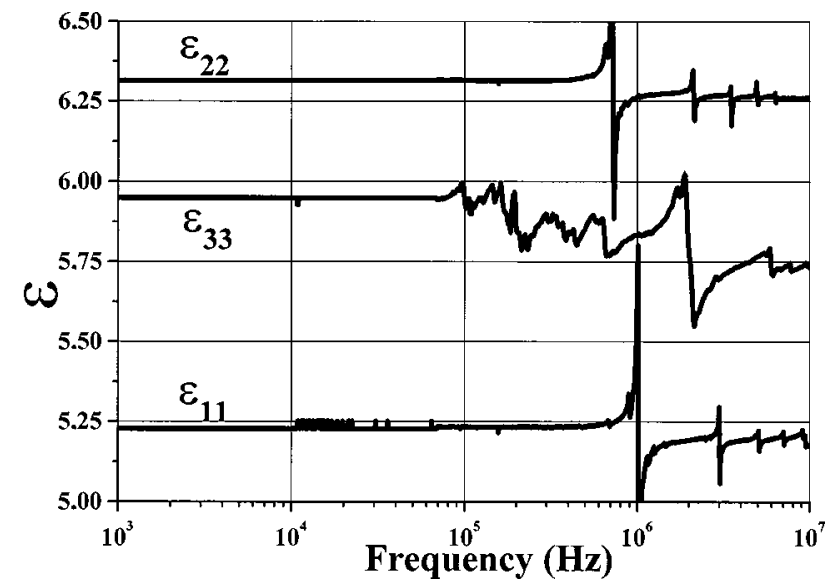

Fig. 2. Frequency dispersion of dielectric permittivities $\epsilon_{11}$, $\epsilon_{22}$, and $\epsilon_{33}$ of 2A5NPDP. 
Table 1. Refractive Indices $n_{i j}$ at $\lambda=632.8 \mathrm{~nm}$. $^{a}$ Dielectric Permittivities $\epsilon_{i j}$ and Electro-Optic Coefficients $r_{i j k}$ of 2A5NPDP at a Frequency of $1 \mathbf{k H z}^{b}$

\begin{tabular}{cccccrr}
\hline$n_{i j}$ & Refractive Index & $\epsilon_{i j}$ & Refractive Index & $r_{i j k}{ }^{T}(\mathrm{pm} / \mathrm{V})$ & Interferometric Setup & Sénarmont's Setup \\
\hline$n_{11}$ & 1.6115 & $\epsilon_{11}$ & 5.25 & $r_{113}\left(r_{13}\right)$ & $1.8 \pm 0.2$ & - \\
$n_{22}$ & 1.6447 & $\epsilon_{22}$ & 6.3 & $r_{223}\left(r_{23}\right)$ & $2 \pm 0.2$ & - \\
$n_{33}$ & 1.7373 & $\epsilon_{33}$ & 5.95 & $r_{333}\left(r_{33}\right)$ & $-6.1 \pm 0.6$ & - \\
& & & $\left|r_{232}\right|\left(\left|r_{42}\right|\right)$ & - & $0.2 \pm 0.04$ \\
& & & $\left|r_{131}\right|\left(\left|r_{51}\right|\right)$ & - & $1.8 \pm 0.3$ \\
& & & $r_{b}$ & $9.2 \pm 1.8$ & $9.5 \pm 1.5$ & -1.2 \\
& & & $r_{c}$ & $-0.3 \pm 0.1$ & $-0.3 \pm 0.04$ \\
\hline
\end{tabular}

${ }^{a}$ Ref. 5

${ }^{b}$ The signs of the EO coefficients have to be considered relative signs. The values of $r_{a}, r_{b}$ and $r_{c}$ that correspond to the interferometric setup are calculated from Eq. (12).

\section{EXPERIMENTAL RESULTS}

We measured the EO coefficients of the 2A5NPDP crystal as well as its dielectric permittivities. Gold electrodes were evaporated onto the surfaces of the sample, which was cut and polished appropriately for its size and geometry. Dielectric measurements were performed with a HP 4092 impedance analyzer with an amplitude voltage of $1 \mathrm{~V}$ and a frequency that varied from $1 \mathrm{kHz}$ to more than $10 \mathrm{MHz}$.

The EO coefficients $r_{113}, r_{223}$, and $r_{333}$ (or, in the abbreviated notation, $r_{13}, r_{23}$, and $r_{33}$ ) were determined under dc voltage by the interferometric setup, whereas $r_{232}$ and $r_{131}\left(r_{42}\right.$ and $\left.r_{51}\right)$ together with the effective $r_{a}, r_{b}$, and $r_{c}$ coefficients (linear combination of $r_{13}, r_{23}$, and $r_{33}$ ) were obtained by the Sénarmont method, mainly at a modulation frequency of $1 \mathrm{kHz}$. Some of these coefficients have large values, and the figures of merit for EO modulation were determined..$^{13}$

Figure 2 displays the frequency dependence of the principal dielectric permittivities $\epsilon_{11}, \epsilon_{22}$, and $\epsilon_{33}$. We can see the anisotropy of these coefficients recorded at $1 \mathrm{kHz}$ $\left(\epsilon_{22}>\epsilon_{33}>\epsilon_{11}\right)$. The small step between the values recorded on both sides of the piezoelectric resonances $(0.05$ for $\epsilon_{11}$ and $\epsilon_{22} ; 0.25$ for $\epsilon_{33}$ ) reveals the relative weakness of the piezoelectric coefficients.

As a consequence, the correction [Eq. (15)] that is due to the elastic deformation of the crystal in the determination of EO coefficients $r_{13}, r_{23}$, and $r_{33}$ can be neglected. These values are listed in Table 1, together with the values of the coefficients derived from the Sénarmont setup by means of Eq. (12). In all these calculations we used the values of the refractive indices recorded in the same crystal. $^{5}$ We can see from the table the fairly good agreement in the effective coefficients $r_{a}, r_{b}$, and $r_{c}$ between the experimental data obtained from the Sénarmont measurements and the values deduced from $r_{13}, r_{23}$ and $r_{33}$ by use of Eq. (8). It can also be seen that the relative sign of the coefficients can be obtained from the change of the electric-field-induced phase shift according to the direction of the $c$ axis.

It is necessary to point out that all values of the $\mathrm{EO}$ coefficients listed in Table 1 were recorded at zero or low frequency of the applied electric field. Therefore they can be related to the total $\mathrm{EO}$ coefficients $r^{T}$ expressed by Eq. (6).

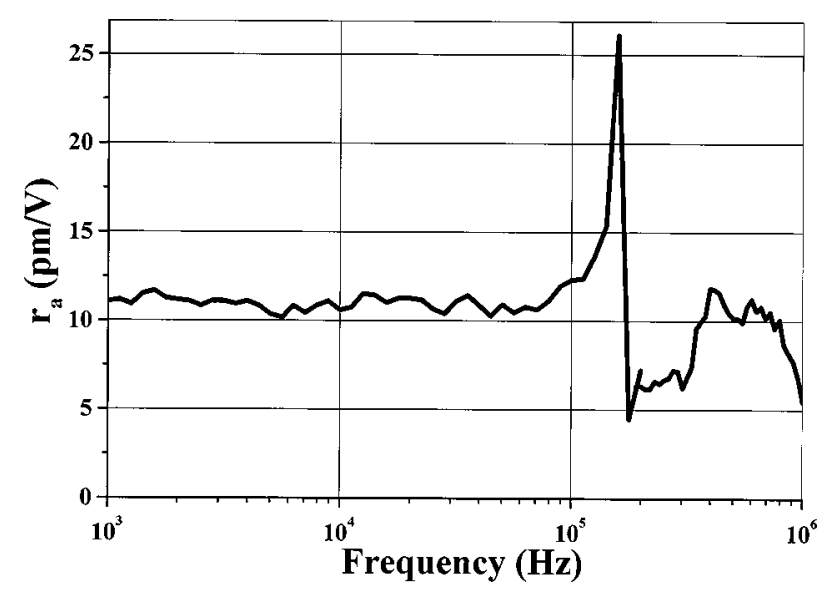

Fig. 3. Frequency dispersion of electro-optic coefficient $r_{a}$ of 2A5NPDP.

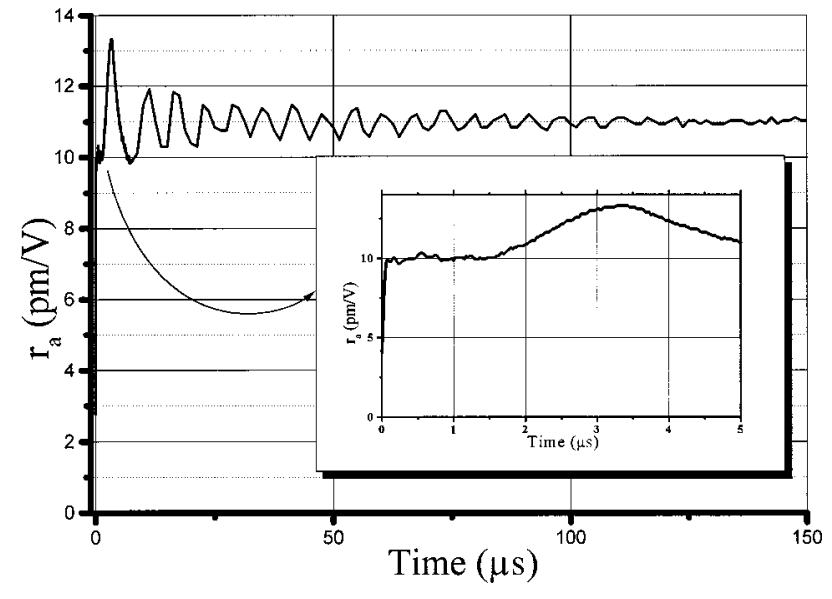

Fig. 4. Time response of electro-optic coefficient $r_{a}$ in 2A5NPDP.

Accordingly, the piezo-optic contribution, if it does exist, can be evaluated from the measurements of the EO coefficients on both sides of the piezoelectric resonances. We therefore recorded the frequency dependence of $r_{a}$ from $1 \mathrm{kHz}$ to $1 \mathrm{MHz}$. The results, as illustrated in Fig. 3 , show that the coefficient remains constant as a function of frequency below the main resonance, lying at $\sim 170$ $\mathrm{kHz}$. As can be seen from the corresponding dielectric 
permittivity $\epsilon_{33}$, many resonances exist up to $10 \mathrm{MHz}$, which implies that additional measurements at higher frequency are needed for reaching the clamped coefficient $r_{a}^{s}$ [according to Eq. (5)]. To make these measurements we investigated the time response of EO coefficient $r_{a}$ to a voltage step, using the Sénarmont setup. The results are plotted in Fig. 4. The value of $r_{a}(11 \mathrm{pm} / \mathrm{V})$ obtained after a long time $(200 \mu \mathrm{s})$ is in quite good agreement with the data measured at $1 \mathrm{kHz}$ (Fig. 3).

In the long-time response range, the output signal oscillates with a period that corresponds to the main piezoelectric frequency. These oscillations disappear in the short-time range, or equivalently in the frequency range above the resonances, leading to a value of $r_{a}{ }^{s}$ that is equal to $10 \mathrm{pm} / \mathrm{V}$.

The piezo-optic contribution to $r_{a}$ is therefore $\sim 10 \%$ of the total value. It is still lower for $r_{b}$ and $r_{c}$, so $r^{T}$ $\approx r^{s}$ for these coefficients. These results are consistent with the dielectric data of Fig. 2, which reveal a small piezoelectric contribution to $r_{a}$.

These clamped values of EO coefficients thus obtained are the sums of the ionic and the electronic contributions. They can be compared with the purely electronic part $r^{e}$, as deduced from the SHG coefficient by Eq. (2).

\section{ESTIMATION OF THE DIFFERENT CONTRIBUTIONS}

The calculated values of $r^{e}$ are listed in Table 2, together with the experimental $r^{T}$ data, which are nearly equal to $r^{s}$ because the piezo-optic contribution is negligible. For the values of $d_{i j k}$ reported in Table 2 and used to calculate the value of $r_{i j k}{ }^{e}$, we have to make the following points: The error in Table 2 is underestimated because only experimental accuracy is taken into account. In fact, a systematic error can also be considered, depending on the method that is used. Indeed, we can see a nonnegligible discrepancy in the values of $d_{113}$ and $d_{223}\left(d_{15}\right.$ and $d_{24}$ in the abbreviated notation) in data reported by Kotler et $a l .{ }^{4}$ and the results given by Feve et al. ${ }^{5}$ Therefore the value of $d_{33}$ and thus that of $r_{33}{ }^{e}$ are certainly overestimated.

A comparison of the $r^{e}$ and $r^{s}\left(=r^{e}+r^{j}\right)$ values shows that modulation of the electrons by the applied electric field is the main component of the Pockels EO effect for

\begin{tabular}{|c|c|c|c|}
\hline \multicolumn{4}{|c|}{$\begin{array}{c}\text { Table 2. Measured Values of } r_{i j k}^{T}-r_{i} \\
\text { Calculated Values of } r_{i j k} \text { [from Eq. (2)], and } \\
\text { Measured Values of } d_{i j k} \text { for } 2 A 5 N P D P\end{array}$} \\
\hline$i, j, k$ & $\begin{array}{l}\text { Measured } \\
r_{i j k}^{T /-/ r_{i j k} s /} \\
\quad(\mathrm{pm} / \mathrm{V})\end{array}$ & $\begin{array}{l}\text { Calculated } \\
\quad / r_{i j k}{ }^{e} / \\
(\mathrm{pm} / \mathrm{V})\end{array}$ & $\begin{array}{l}\text { Measured } \\
\quad / d_{i j k} / \\
(\mathrm{pm} / \mathrm{V})\end{array}$ \\
\hline $1,1,3$ & $1.8 \pm 0.2$ & $1.6 \pm 0.3$ & $2 \pm 0.2$ \\
\hline $2,2,3$ & $2 \pm 0.2$ & $0.25 \pm 0.05$ & $0.4 \pm 0.08$ \\
\hline $3,3,3$ & $6.1 \pm 0.6$ & $8 \pm 1.5$ & $12 \pm 1$ \\
\hline $2,3,2(24)$ & $0.2 \pm 0.04$ & $0.25 \pm 0.05$ & $0.4 \pm 0.08$ \\
\hline $1,3,1(15)$ & $1.8 \pm 0.3$ & $1.2 \pm 0.25$ & $2 \pm 0.2$ \\
\hline
\end{tabular}

${ }^{a} d_{33}$ was measured relative to quartz $\left(d_{11}=0.4 \mathrm{pm} / \mathrm{V}\right)$ for $\lambda$ $=1074 \mathrm{~nm},{ }^{4}$ and $d_{24}$ and $d_{15}$ were measured relative to KTP $\left(d_{24}=2.65 \mathrm{pm} / \mathrm{V}\right.$ for $\lambda=1118 \mathrm{~nm}$ and $d_{15}=1.4 \mathrm{pm} / \mathrm{V}$ for $\left.\lambda=1340 \mathrm{~nm}\right) .^{5}$ all coefficients except the coefficient $r_{23}$. We note also the large anisotropy in $r^{e}$ and $r^{s}$ values, which we attempt to interpret in what follows.

The electronic coefficient $r^{e}{ }_{33}$ is indeed much larger than the corresponding values of $r_{13}^{e}, r_{23}^{e}, r_{42}^{e}$, and $r^{e}{ }_{51}$. We can understand by this considering the relative angle between, on the one hand, the light-polarization axis involved for a given coefficient ( $r$ or $d$ ) and, on the other hand, the preferential direction of a molecule's charge-transfer axis. This angle is much more favorable to coefficient $r^{e}{ }_{33}$, for which the impinging light is polarized along the $c$ axis and thus forms a small angle $\left(\sim 35^{\circ}\right)$ with respect to the organic molecule's charge-transfer axis and yields the largest value. Under the same conditions, the value of EO coefficient $r^{e}{ }_{13}$ (impinging light polarized along the $a$ axis, which forms an angle of $\sim 61^{\circ}$ with respect to the molecule's charge-transfer axis) is, as listed in Table 2, larger than that of $r^{e}{ }_{23}$ (impinging light polarized along the $b$ axis, which forms an angle of $\sim 71^{\circ}$ with respect to the charge-transfer axis). Likewise, we can deduce that the value of $r^{e}{ }_{51}\left(r^{e}{ }_{131}\right)$ is larger than that of $r_{42}^{e}\left(r_{232}^{e}\right)$ but smaller than that of $r^{e}{ }_{13}$.

As was mentioned above, the electronic contributions predominate in all the EO coefficients except $r_{23}$, for which the ionic part is larger (see Table 2). This difference could be related to the larger value of permittivity $\epsilon_{22}$ (see Table 1), which reveals higher polarizability of the inorganic groups along the $b$ axis that could induce a larger ionic contribution to EO coefficient $r_{23}$.

\section{SUMMARY}

We measured the EO coefficients of 2A5NPDP crystals at low and high frequencies. These results were compared with the purely electronic contribution made by the SHG coefficients. A lattice deformation and changes in ionic polarizabilities are expected because of the mineral group, leading to a low-frequency dispersion of the quadratic $\chi^{(2)}$ or EO coefficient. In fact, the magnitudes of these coefficients, except $r_{23}$, are mainly electronic in origin, and the piezo-optic contribution caused by the deformation is smaller than $10 \%$. The electronic EO or SHG coefficients are highly anisotropic and are due mainly to organic components; they are interpreted as being due to the charge-transfer transition of the push-pull molecules.

In conclusion, whereas they have stronger resistance to mechanical and optical damage, the bounds of the mineral $\left[\mathrm{H}_{2} \mathrm{PO}_{4}\right]_{n}$ layers to the organic component do not strongly affect the EO and SHG properties of 2A5NPDP.

\section{ACKNOWLEDGMENTS}

We are grateful to the Groupement de Recherche 1181 "Matériaux pour l'Optique Nonlinéaire" of the Centre National de la Recherche Scientifique for its support of this study.

J. Zaccaro's e-mail address is zaccaro@polycnrs-gre.fr; that of J. P. Salvestrini is salvestr@ese-metz.fr; that of A. Ibanez is ibanez@polycnrs-gre.fr; that of M. D. Fontana is fontana@ese-metz.fr. 


\section{REFERENCES}

1. D. S. Chemla and J. Zyss, eds., Nonlinear Optical Properties of Organic Molecules and Crystals (Academic, Orlando, Fla., 1987).

2. P. Günter, ed., Electrooptic and Photorefractive Materials (Springer-Verlag, Berlin, 1987).

3. J. Pécaut and R. Masse, "Structure of bis(2-amino-5nitropyridinium) dichromate as a step towards the design of efficient organic-inorganic non-linear optical crystals," Acta Crystallogr., Sect. B: Struct. Sci. 49, 277-282 (1993).

4. Z. Kotler, R. Hierle, D. Josse, J. Zyss, and R. Masse, "Quadratic nonlinear-optical properties of a new transparent and highly efficient organic-inorganic crystal: 2-amino-5nitropyridium-dihydrogen phosphate (2A5NPDP)," J. Opt. Soc. Am. B 9, 534-547 (1992); S. Khodja, D. Josse, and J. Zyss, "Near-infrared optical parametric oscillation in an organomineral crystal," J. Opt. Soc. Am. B 15, 751-758 (1998).

5. J. P. Feve, B. Boulanger, I. Rousseau, G. Marnier, J. Zaccaro, and A. Ibanez, "Second-harmonic generation properties of 2-amino-5-nitropyridinium-dihydrogenoarsenate and dihydrogenophosphate organic-inorganic crystals," IEEE J. Quantum Electron. 35, 66-71 (1999).

6. M. Sigelles and R. Hierle, "Determination of the electrooptic coefficients of 3-methyl 4-nitropyridine 1-oxyde by an interferometric phase modulation technique," J. Appl. Phys. 52, 4199-4204 (1981).

7. J. P. Salvestrini, M. D. Fontana, B. Wyncke, and F. Brehat, "Comparative measurements of the frequency dependence of electrooptical and dielectric coefficients in inorganic crystals," Nonlinear Opt. 17, 271-280 (1997).
8. A. Ibanez, J. P. Levy, C. Mouget, and E. Prieur, "Crystal growth of a promising nonlinear optical material: 2-amino-5-nitropyridinium-chloride," J. Solid State Chem. 129, 22-29 (1997).

9. J. Zaccaro, M. Bagieu-Beucher, J. Espeso, and A. Ibanez, "Structural characterization and crystal growth of the 2amino-5-nitropyridinium-dihydrogenophosphate/arsenate hybrid solid solution," J. Cryst. Growth 186, 224-232 (1998); J. Zaccaro, B. Capelle, and A. Ibanez, "Crystal growth of hybrid nonlinear optical materials: 2-amino-5nitropyridinium-dihydrogenoarsenate and dihydrogenophosphate," J. Cryst. Growth 180, 229-237 (1997).

10. R. Masse and J. Zyss, "A new approach in the design of polar crystals for quadratic nonlinear optics exemplified by the synthesis of 2-amino-5-nitropyridium-dihydrogen monophosphate (2A5NPDP)," Mol. Eng. 1, 141-152 (1991); J. Pecaut, Y. Lefur, and R. Masse, "Crystal engineering and structural investigations of the 2-amino-5-nitropyridinium salts $\mathrm{C}_{5} \mathrm{H}_{6} \mathrm{~N}_{3} \mathrm{O}_{2}{ }^{+} \cdot \mathrm{HSO}_{4}^{-}$and $\mathrm{C}_{5} \mathrm{H}_{6} \mathrm{~N}_{3} \mathrm{O}_{2}{ }^{+} \cdot \mathrm{H}_{2} \mathrm{AsO}_{4}{ }^{-1}$," Acta Crystallogr., Sect. B: Struct. Sci. 49, 535-541 (1993).

11. R. Morita and P. V. Vidakovic, "Angle and temperature tuning of phase-matched second-harmonic generation in $N$-(4-nitrophenyl)- $N$-methylaminoacetonitrile," Appl. Phys. Lett. 61, 2854-2856 (1992).

12. M. Aillerie, M. D. Fontana, F. Abdi, C. Carabatos-Nedelec, N. Theofanous, and G. Alexakis, "Influence of the temperature-dependent spontaneous birefringence in the electro-optic measurements of $\mathrm{LiNbO}_{3}$," J. Appl. Phys. 65, 2406-2408 (1989)

13. J. P. Salvestrini, J. Zaccaro, A. Ibanez, and M. D. Fontana, "Investigation of electrooptic modulation from organicinorganic crystals," Appl. Phys. B 67, 761-763 (1998). 